\title{
Efecto de la carga laboral y la rotación del personal en la productividad de los servidores judiciales en el Consejo de la Judicatura de Los Ríos, Ecuador en el año 2018
}

\author{
Effect of workload and staff turnover on the productivity of judicial servants in \\ the Judicial Council of Los Ríos, Ecuador in 2018
}

Gisella Cecibel Maliza Cerezo. ${ }^{1} \&$ Carlos Alberto Bastidas Vaca. ${ }^{2}$

Recibido: 23-05-2019 / Revisado: 10-06-2019 /Aceptado: 11-07-2019/ Publicado: 06-09-2019

\begin{abstract}
DOI: $\underline{\text { https://doi.org/10.33262/cienciadigital.v3i3.3.768 }}$

The judicial system which is part of the five powers of the Ecuadorian state, and through which the documentary study and implementation of this article has been generated, through which we will refer to the Judicial Council of the province of Los Ríos-Ecuador In 2018, one of the public sector entities that is going through serious budgetary problems generating study variables, these being the independent variables such as workload and staff turnover in this judicial entity and its effect on the productivity of These public servants, frames that the analysis of these variables, the study of them and the respective recommendations is very important in order to achieve greater productivity, leveraged in the efficiency and effectiveness that these justice operators must perform, seeking the aim to improve productivity and obtain positive results by implementing time load and rotation Ion of the personnel in the different areas of this institution, which seeks the full purpose of satisfying the needs of the Ecuadorian people and especially of this population that require justice operators at different stages or procedural causes, with continuous improvement prevailing planning of the study variables with the sole objective of improving the productivity of judicial management, and being able to frame ourselves in the good and timely quality of the service, in order to mitigate the labor risks that may occur in the course and development of activities labor lawsuits and obtain as a result a quality public management.
\end{abstract}

${ }^{1}$ Función Judicial, gisella.maliza@funcionjudicial.gob.ec

2 Universidad Estatal de Milagro, cbastivasv@unemi.edu.ec 
Referring to the importance of planning in terms of the workload and turnover of staff in the Judicial Council of the province of the Babahoyo, Ecuador.

Any job requires more or less physical or mental effort, determining then that when changes in production processes or work teams are anticipated, it is very important to plan training plans in advance. The company's prevention plan should not obviate this reality and both the risk assessment and the planning of preventive activities should reflect the health risks related to the physical and mental burden of work that entails affecting the productivity of the judicial public servant

Keywords: Workload, staff turnover, productivity.

\section{Resumen}

El sistema judicial el cual forma parte de los cinco poderes del estado ecuatoriano, y por medio del cual se ha generado el estudio documental y realización del presente artículo, mediante el cual haremos referencia al Consejo de la Judicatura de la provincia de los RíosEcuador en el año 2018, una de las entidades del sector público que está atravesando serios problemas presupuestarios generando unas variables de estudio, siendo estas las variables independientes como son la carga laboral y la rotación del personal en esta entidad judicial y su efecto en la productividad de estos servidores públicos, enmarca que es muy importante el análisis de estas variables, el estudio de las mismas y las recomendaciones respectivas con el fin de lograr una mayor productividad, apalancados en la eficiencia y eficacia que estos operadores de justicia lo deben realizar, buscando el objetivo de mejorar la productividad y obtener resultados positivos al implementar la carga horaria y la rotación del personal en las diferentes áreas de esta institución, que busca el fin pleno de satisfacer las necesidades del pueblo ecuatoriano y en especial de esta población que requieren de los operadores de justicia en las diferentes etapas o causas procesales, prevaleciendo la mejora continua la planificación de las variables de estudio con el único objetivo de mejorar la productividad de la gestión judicial, y poder enmarcarnos en la buena y oportuna calidad del servicio, para de esta forma mitigar los riesgos laborales que se pueden dar en el transcurso y desarrollo de las actividades judiciales laborales y obtener como resultado una gestión pública de calidad.

Haciendo referencia a la importancia de la planificación en cuanto a la carga horaria y rotación del personal en el Consejo de la Judicatura de la provincia de Los Ríos, Babahoyo, Ecuador.

Cualquier trabajo exige en mayor o menor medida un esfuerzo físico o mental, determinando entonces que cuando se prevé cambios en los procesos productivos o en los equipos de trabajo, es muy importante planificar con antelación planes de formación. El plan de prevención de la empresa no debe obviar esta realidad y tanto en la evaluación de riesgos como en la planificación de las actividades preventivas debe reflejarse los riesgos para la salud relacionada con la carga física y mental del trabajo que conlleva a afectar la productividad del servidor público judicial.

Palabras clave: Carga laboral, rotación de personal, productividad.

\section{Introducción}

El Consejo de la Judicatura ubicado en la provincia de Los Ríos, Ecuador en la actualidad es una de las instituciones del sector público, que está atravesando un grave problema presupuestario que está afectando en muchas formas al desempeño laboral que implicado directamente en la rotación 
del personal y su carga laboral motivo de estudio y desarrollo de este artículo. Y que de una u otra forma va afectar la imagen institucional y por ende a la productividad de estos funcionarios.

\section{CONSEJO DE LA JUDICATURA DE LOS RIOS}

\section{Distributivo de la provincia actualizado}

Hasta la presente fecha la Dirección Provincial de Los Ríos se encuentra conformada por un total de 420 servidores jurisdiccionales y administrativos, los cuales se detallan a continuación:

Tabla 1. Modalidad de Vinculación

\begin{tabular}{lcc}
\multicolumn{1}{c}{$\frac{\text { MODALIDAD DE }}{\text { VINCULACION }}$} & $\begin{array}{c}\text { No. de } \\
\text { funcionarios }\end{array}$ & Porcentaje \% \\
\hline Contratos & 10 & 2,38 \\
Nombramientos Definitivo & 154 & 36,66 \\
Nombramientos & 256 & 60,95 \\
Provisionales & $\mathbf{4 2 0}$ & $\mathbf{1 0 0 \%}$ \\
\hline Total & &
\end{tabular}

Debido al déficit presupuestario las siguientes vacantes y cargos no se encuentran financiadas, más sin embargo representan piezas claves para que el manejo administrativo y jurisdiccional de la Dirección Provincial del Consejo de la Judicatura de Los Ríos, pueda desarrollarse de una manera eficiente y eficaz en servicio de la ciudadanía, motivo por el cual la Dirección Provincial de Los Ríos se ha visto obligado a encargar estas coordinaciones a otros funcionarios judiciales, sobrecargándolos de esta manera de nuevas funciones:

Tabla 2. Modalidad de Vinculación

\begin{tabular}{|c|c|c|c|}
\hline \multicolumn{4}{|c|}{ Órgano administrativo } \\
\hline No. & Denominación del cargo & Dependencia & $\begin{array}{c}\text { Modalidad } \\
\text { (nombramiento } \\
\text { provisional/ contrato) }\end{array}$ \\
\hline 1 & $\begin{array}{l}\text { Coordinador provincial de talento } \\
\text { humano }\end{array}$ & Unidad provincial de talento humano & Nombramiento provisional \\
\hline 2 & $\begin{array}{l}\text { Coordinador de secretaria } \\
\text { provincial y archivo }\end{array}$ & Secretaria provincial y archivo & Contrato \\
\hline 3 & Coordinador de gestión procesal & Gestión procesal & Nombramiento provisional \\
\hline 4 & $\begin{array}{l}\text { Coordinador provincial de } \\
\text { planificación }\end{array}$ & Unidad de planificación & $\begin{array}{l}\text { Contrato - nunca ha sido } \\
\text { cubierta }\end{array}$ \\
\hline 5 & Coordinador provincial financiero & Unidad provincial financiera & Nombramiento provisional \\
\hline 6 & Coordinador provincial tics & Unidad provincial tics & Necesidad de creación \\
\hline 7 & $\begin{array}{l}\text { Coordinador provincial de justicia } \\
\text { de paz }\end{array}$ & Unidad provincial de justicia de paz & Necesidad de creación \\
\hline
\end{tabular}


ISSN: 2602-8085

\begin{tabular}{|c|c|c|c|}
\hline 8 & $\begin{array}{l}\text { Técnico de secretaria provincial y } \\
\text { archivo }\end{array}$ & Secretaria provincial y archivo & Nombramiento provisional \\
\hline 9 & Asistente administrativo & Dirección provincial de Los Ríos & Contrato \\
\hline 10 & Analista 2 & Unidad provincial administrativa & Contrato \\
\hline 11 & Gestor de archivo & $\begin{array}{l}\text { Unidad judicial de la familia, mujer, } \\
\text { niñez y adolescencia en Quevedo }\end{array}$ & Nombramiento provisional \\
\hline 12 & $\begin{array}{l}\text { Técnico de ventanilla e } \\
\text { información }\end{array}$ & Sala multicompetente de Quevedo & Nombramiento provisional \\
\hline 13 & Trabajadora social & $\begin{array}{l}\text { Unidad judicial de la familia, mujer, } \\
\text { niñez y adolescencia en Babahoyo }\end{array}$ & Nombramiento \\
\hline 14 & Jueza provincial & Sala multicompetente de Babahoyo & Nombramiento \\
\hline 15 & Abogado provincial 2 & Unidad provincial de asesoría jurídico & Contrato \\
\hline 16 & Técnico & $\begin{array}{l}\text { Unidad provincial de comunicación } \\
\text { social }\end{array}$ & Nombramiento provisional \\
\hline 17 & $\begin{array}{l}\text { Coordinador provincial de talento } \\
\text { humano }\end{array}$ & Unidad provincial de talento humano & Nombramiento provisional \\
\hline 18 & $\begin{array}{l}\text { Secretario (a) de juzgado y } \\
\text { unidades judiciales }\end{array}$ & Unidad judicial de lo civil & Nombramiento provisional \\
\hline 19 & Técnico provincial administrativo & Unidad provincial administrativa & Nombramiento provisional \\
\hline 20 & Coordinador de asesoría jurídica & Unidad provincial asesoría jurídica & Contrato \\
\hline 21 & Jueza & $\begin{array}{l}\text { Unidad judicial multicompetente de } \\
\text { Mocache }\end{array}$ & Nombramiento \\
\hline 22 & Técnico provincial administrativo & Unidad provincial administrativa & Nombramiento provisional \\
\hline 23 & Liquidador - pagador & Unidad judicial multicompetente & Nombramiento provisional \\
\hline 24 & Ayudante judicial & $\begin{array}{l}\text { Unidad judicial primera de la familia, } \\
\text { mujer, niñez y adolescencia }\end{array}$ & Nombramiento provisional \\
\hline 25 & $\begin{array}{l}\text { Coordinador de secretaria } \\
\text { provincial y archivo }\end{array}$ & Secretaria provincial y archivo & Contrato \\
\hline 26 & Coordinador de gestión procesal & Gestión procesal & Contrato \\
\hline 27 & Mediador coordinador de oficina & Servicio de mediación & Contrato \\
\hline 28 & Gestor de archivo & $\begin{array}{l}\text { Sala multicompetente de la corte } \\
\text { provincial de justicia }\end{array}$ & Contrato \\
\hline 29 & Gestor de archivo & $\begin{array}{l}\text { Sala multicompetente de la corte } \\
\text { provincial de justicia }\end{array}$ & Contrato \\
\hline 30 & $\begin{array}{l}\text { Técnico de ventanilla e } \\
\text { información }\end{array}$ & Unidad judicial de lo civil y mercantil & Contrato \\
\hline 31 & $\begin{array}{l}\text { Técnico de ventanilla e } \\
\text { información }\end{array}$ & Unidad judicial de lo civil & Contrato \\
\hline 32 & $\begin{array}{l}\text { Técnico de ventanilla e } \\
\text { información }\end{array}$ & Unidad judicial de trabajo & Contrato \\
\hline 33 & $\begin{array}{l}\text { Técnico de ventanilla e } \\
\text { información }\end{array}$ & $\begin{array}{l}\text { Unidad judicial de la familia, mujer, } \\
\text { niñez y adolescencia }\end{array}$ & Contrato \\
\hline 34 & Gestor de archivo & $\begin{array}{l}\text { Unidad judicial de la familia, mujer, } \\
\text { niñez y adolescencia }\end{array}$ & Contrato \\
\hline 35 & $\begin{array}{l}\text { Técnico de ventanilla e } \\
\text { información }\end{array}$ & $\begin{array}{l}\text { Unidad judicial de la familia, mujer, } \\
\text { niñez y adolescencia }\end{array}$ & Contrato \\
\hline 36 & $\begin{array}{l}\text { Técnico de ventanilla e } \\
\text { información }\end{array}$ & $\begin{array}{l}\text { Unidad judicial de la familia, mujer, } \\
\text { niñez y adolescencia }\end{array}$ & Contrato \\
\hline
\end{tabular}




\begin{tabular}{|c|l|l|l|}
37 & Jueza & $\begin{array}{l}\text { Unidad judicial multicompetente de } \\
\text { Vinces }\end{array}$ & Nombramiento \\
\hline 38 & $\begin{array}{l}\text { Técnico de ventanilla e } \\
\text { información }\end{array}$ & $\begin{array}{l}\text { Unidad judicial de la familia, mujer, } \\
\text { niñez y adolescencia }\end{array}$ & Nombramiento provisional \\
\hline 39 & $\begin{array}{l}\text { Secretario (a) de juzgado y } \\
\text { unidades judiciales }\end{array}$ & Unidad judicial multicompetente & Nombramiento provisional \\
\hline 40 & $\begin{array}{l}\text { Técnico de secretaria provincial y } \\
\text { archivo }\end{array}$ & Secretaria provincial y archivo & Nombramiento provisional \\
\hline 41 & Médico perito & Unidad judicial penal de Babahoyo & Nombramiento provisional \\
\hline 42 & Ayudante judicial & Unidad judicial penal de Quevedo & Nombramiento provisional \\
\hline 43 & Notificador - citador & Unidad judicial civil de Babahoyo & Nombramiento provisional \\
\hline 44 & Notificador - citador & $\begin{array}{l}\text { Unidad judicial multicompetente de } \\
\text { Vinces }\end{array}$ & Nombramiento provisional \\
\hline 45 & Ayudante judicial & $\begin{array}{l}\text { Unidad judicial de la familia, mujer, } \\
\text { niñez y adolescencia }\end{array}$ & Nombramiento provisional \\
\hline
\end{tabular}

El cuadro y en resumen la información antes mencionada hace hincapié y forma parte del insumo para el desarrollo de este artículo.

Según (Polo Vargas, Santiago Briñez, Navarro Segura, \& Alí Nieto, 2016). Una de las esferas más significativas en la vida de las personas es el trabajo, porque contribuye con su subsistencia y trascendencia. Por ello, (Zenteno Hidalgo \& Dúran Silva, 2016) manifiestan que los resultados positivos de la empresa están ligados a la gestión del recurso humano y su contexto; ya que el trabajo se transforma en un compromiso psicológico, físico, emocional y social en la realización de las actividades cotidianas. Esto explica por qué, cuando la relación hombre-trabajo se altera, llegan a producirse perjuicios con desenlaces psicopatológicos que inciden en la producción organizacional y van a afectar la imagen de la misma.

En referencia a lo antes mencionado y lo que está sucediendo en la actualidad la carga laboral y la rotación de personal son dos variables que se encuentran presente en las instituciones públicas, teniendo efectos comunes en cada una de ellas y en especial en el Consejo de la Judicatura de los Ríos. El recurso humano parte esencial en el desarrollo de toda empresa, permite que las instituciones sean productivas, por lo cual resulta indispensable la presencia y compromiso del empleado para el cumplimiento de los objetivos institucionales. Y a la vez el compromiso institucional de velar por el bienestar de sus trabajadores.

La rotación de personal en la actualidad se ha convertido en un fenómeno creciente en las instituciones del sector público que brindan servicios, y en este caso en el sector de la función judicial. Lo que genera a las organizaciones enfrentarse a problemas de adaptación del nuevo personal a las exigencias del trabajo; y afectación directa en el ciclo productivo y el grupo de trabajo conformado para una actividad en común. Obteniendo como resultado insatisfacción en los clientes, dando una ventana de observación negativa de la institución.

La rotación de personal es uno de los fenómenos laborales que impactan negativamente a la productividad de las naciones y en algunos sectores de la economía, representa la pérdida de recursos financieros y humanos que no son fácilmente recuperables porque implica renovar esfuerzos de ciclos de capacitación, curvas prolongadas de aprendizaje y en ese camino, el desperdicio de recursos técnicos e insumos. (Hernández Chávez, Hernández Chávez, \& Mendieta Ramírez , 2013) 
El presente artículo tiene como objetivo principal determinar la relación existente entre la carga laboral, rotación de personal y la productividad de los servidores judiciales del Consejo de la Judicatura de la Provincia de Los Ríos, Ecuador.

La relevancia de este análisis implica en que permite conocer la magnitud de la relación que guardan estas tres variables de estudios y su injerencia en la productividad y satisfacción laboral de los servidores judiciales y en especial los de la provincia de los Ríos; por lo tanto, puede ser una herramienta para generar estrategias, el diseño e implementación de políticas públicas laborales con el fin único de buscar la satisfacción plena de los clientes.

El presente documento está compuesto por cuatro apartados, en el primero se plantea la problemática general del estudio. En segundo lugar, se describe la fuente de información utilizada en el estudio. Posteriormente, se realiza un análisis documental, descriptivo - correlacional de la relación existente entre las variables carga laboral, rotación de personal y productividad de los servidores judiciales. Finalmente, el último apartado cubre las conclusiones del estudio.

\section{Desarrollo}

La Constitución de la República del Ecuador de 2008 reconoce al trabajo como una "fuente de felicidad y de realización personal necesaria en la consecución de una vida plena, además de mecanismo de integración social" (SENPLADES, 2013). De este modo, la evaluación del trabajo no debe realizarse únicamente por la cantidad sino por la calidad del mismo. El trabajo es un derecho de los ciudadanos, se encuentra garantizado en la constitución de la república y constituye uno de los deberes más altos del estado garantizar todos estos derechos.

El art. 326 de la Constitución de la República del Ecuador de 2008, sustenta un principio importante "Toda persona tendrá derecho a desarrollar sus labores en un ambiente adecuado y propicio, que garantice su salud, integridad, seguridad, higiene y bienestar". Los empleadores están obligados a garantizar al trabajador un ambiente adecuado que le brinde bienestar y le permita aportar a la institución pública y a la sociedad misma.

La rotación de personal está influenciada en gran parte por la insatisfacción y motivación laboral. Lo que presume que la salida del personal se relaciones a temas exclusivamente laborales y en algunos de los casos pueden ser detectados mediante un estudio de fluctuación potencial, pues si se aplican las medidas correctivas necesarias se puede reducir el índice de rotación.

Otras de las variables que afecta sin duda alguna la productividad de los trabajadores es la carga laboral o exceso de trabajo; el mismo que puede ser físico o mental y que se viene desarrollando en las instituciones públicas. Según (Ceballos Vásquez, Rolo González, Hérnandez Fernaud, Díaz Cabrera, Paravic Klijn, \& Burgos Moreno, 2015) afirma:

La carga mental es un constructo multidimensional definido como la interacción entre las demandas cognitivas de la tarea (por ejemplo, memoria y atención), las características de la persona (por ejemplo, nivel educativo y autoeficacia) y las características de la situación (por ejemplo, presión temporal). Entre sus causas cabe citar características de la tarea (por ejemplo, demandas de memoria y atención), presiones temporales y ritmo de trabajo, así como, las funciones a desempeñar, el grado de autonomía, y la interacción con otras personas. (p. 316)

El desequilibrio entre las demandas de la tarea y las capacidades y características de los trabajadores puede provocar sobrecarga o subcarga mental de trabajo (Ceballos Vásquez, Rolo González, Hérnandez Fernaud, Díaz Cabrera, Paravic Klijn, \& Burgos Moreno, 2015). En referencia a este aporte se puede decir que si va afectar la rotación laboral y la carga laboral de la mima en el desarrollo de las actividades cotidianas en este sector además de la productividad de estos actores públicos. 
Por otra parte, O’Donell y Eggemeier citado por (González Muñoz \& Gutiérrez Mártinez, 2006) afirma: "El término de carga de trabajo, se refiere a qué parte de la capacidad limitada del operador es requerida para desarrollar una tarea particular". Es así que, la dificultad para la realización de las tareas principales incrementa los requerimientos de recursos para el procesamiento mental, y si las demandas del procesamiento de una tarea o tareas exceden la capacidad existente, se tendrá como consecuencia, aparte del estrés laboral, un decremento en el desempeño personal y organizacional (González Muñoz \& Gutiérrez Mártinez, 2006). El exceso de trabajo o carga laboral ha generado la necesidad de los empleados de buscar nuevos trabajos que de cierto modo les proporcione estabilidad en su bienestar emocional, mental, físico y satisfacción personal. Motivo por el cual su productividad se ve afectada y por ende la imagen y gestión institucional.

El exceso de trabajo provoca que los empleados se encuentren sometidos a estrés laboral que afecta su productividad dentro de la institución e influye en la decisión de abandono de sus puestos de trabajo para evitar daños en la salud del trabajador. "Una patología moderna derivada del estrés crónico laboral al que se exponen los trabajadores con motivo de su trabajo es el síndrome de Burnout" (Aranda, Pando y Salazar, 2016, p. 219). Freudenberger (citado por Aranda et al., 2016) indica "Burnout es un término anglosajón que significa estar quemado, desgastado, exhausto y perder la ilusión por el trabajo" (p. 219).

El síndrome de Burnout (SB) se define como la sensación de agotamiento, decepción y pérdida de interés por la actividad laboral. Surge especialmente en aquellos que se dedican a profesiones de servicio, como consecuencia del contacto diario con otras personas en su trabajo (Vargas, Niño y Acosta, 2017). "Este síndrome lleva consigo problemas de salud mental, ansiedad, depresión, entre otros" (Vargas et al.,2017, p.114).

Según Quiceno (citado por Vargas et al.,2017): “el Síndrome de Burnout es una respuesta al estrés laboral crónico, acompañado de una experiencia subjetiva de sentimientos, cogniciones y actitudes que provocan alteraciones psicofisiológicas en la persona y consecuencias negativas para las instituciones laborales" (p-114). Motivo por el cual y bajo este análisis es muy importante el desarrollo y estudio de este tema además de cuidar la salud de los trabajadores.

"El Síndrome de Burnout repercute sobre la sociedad en general y es considerado un problema social y de salud pública debido a que genera aumento de los costos económicos y sociales, afecta la moral, el bienestar psicológico, físico y mental de los trabajadores". "Con sus consecuentes implicaciones como ausentismo, rotación, disminución de la productividad y de la calidad" (Vargas et al.,2017, p.114).

Allen, Herst, Bruck \& Sutton (como se citó en Rodríguez y Dabos, 2017) menciona:

Los estudios realizados en las últimas décadas revelan que, si un individuo no alcanza un adecuado equilibrio entre el trabajo y la vida personal, se producen conflictos entre ambos dominios que afectan el desempeño en el trabajo, a partir de la disminución del rendimiento a nivel individual y grupal, el aumento en los índices de rotación y de ausentismo, la insatisfacción laboral y hasta el deterioro en la salud de los individuos, con frecuentes episodios de agotamiento y estrés. (p. 221), motivo que puede llevar a la pérdida del servidor judicial.

Un concepto estrechamente asociado a las exigencias mentales de los puestos de trabajo es el de carga mental, que se define como el esfuerzo cognitivo que debe realizar una persona en un tiempo concreto para hacer frente a determinada cantidad y tipos de tareas (Díaz, Fernández \& Rolo, 2012; Ferrer \& Dalmau, 2014; Gaillard, 2001). El servidor público judicial día a día enfrenta diferentes casos de análisis que exige el estar preparado para actuar en forma eficiente y eficaz por lo que apalancado en este autor se puede determinar la importancia de la carga mental y relacionarla con 
la carga horaria en el desempeño de sus funciones y pretender con este estudio hacer conciencia de la importancia y el cuidado de la salud publica laboral.

Las exigencias mentales del trabajo, tanto por exceso como por defecto, tienen importantes consecuencias en el bienestar y la salud de las personas, así como sobre el logro de los objetivos de la organización, de ahí la relevancia de que sean evaluadas en los contextos de trabajo. (Díaz, Hernández, \& Rolo, 2012). O sea que los logros de los objetivos tienen una relación directa con la carga laboral tomar en cuenta que estos van afectar a la productividad.

Hacker (citado por Ceballos et al., 2016) plantea:

Dos grandes enfoques de análisis de la carga mental de trabajo. El primer enfoque, denominado exigencias recursos, se centra en los procesos cognitivos atencionales sin considerar la interacción entre las demandas de la tarea y los recursos del trabajador. En el segundo enfoque, denominado exigencias de la tarea, se considera la interacción entre las características de la tarea, del trabajador y del contexto. (p.262). Parte importante para el análisis y toma de decisiones.

El desequilibrio que genera el desorden en la asignación de tareas puede producir dos niveles de carga mental: sobrecarga o subcarga. La primera corresponde a la presión mental en la cual se encuentra sometido el trabajador, excediendo de su capacidad de dar respuesta. El segundo nivel hace referencia a la escasa carga de tareas y por ende existe un bajo nivel de exigencia mental. En todo caso las situaciones extremas de carga mental traen consigo algunos riesgos psicológicos como estrés, síndrome de quemarse por el trabajo o Mobbing (Meseguer, et al., 2013). Convirtiéndose de una u otra forma en un acoso laboral que puede llevar a problemas legales Existe una relación entre la carga de trabajo y la rotación de personal que afecta directamente a la productividad de la institución; cuyo impacto de la gestión del recurso humano se ve influenciado por el clima laboral imperante en la organización y factores como la cultura, tanto de la organización como de la industria y el país (Zenteno y Duran, 2016). Según la explicación del autor motiva más el análisis y relación de estudio del tema a tratar que afecte directamente la productividad además ya motiva a la creación de un clima laboral óptimo y adecuado para todos sus empleados.

Una de las propuestas que se ha planteado para controlar las variables principales de esta investigación carga laboral, rotación de personal, productividad y superar las situaciones de desmotivación y bajo compromiso es la implementación de prácticas de recursos humanos de alto desempeño (PAR) que, cuando son establecidas en conjunto, generan sinergia y son la base para generar compromiso, motivar a las personas (Ahmad y Schroeder, 2003). "Las PAR son conceptos, prácticas, variables, ideas y componentes que en su conjunto logran comprometer y empoderar a la fuerza laboral de una organización con alto desempeño" (Moreno, 2011; Tamkin, 2004). Con esto lograríamos conseguir los objetivos propuestos por la institución, pero con el objetivo único de ganar-ganar.

Las medidas de desempeño de las PAR son las consecuencias benéficas de su implementación. Algunas son fácilmente cuantificables; por ejemplo, la valorización financiera de la empresa, rendimiento o productividad de la organización, menor ausentismo y rotación del personal, ventas, ganancias, calidad de productos, reducción de fallas (Zenteno y Duran, 2016).

Podemos decir que bajo el apalancamiento y estudio por parte de este autor, Ahmad y Schroeder (citado por Zenteno y Duran, 2016) sostienen que "la evidencia entre la relación de una adecuada gestión de personas y el desempeño de la organización ha llevado a que varios autores no duden en calificar un recurso humano administrado adecuadamente como un activo valioso e, incluso, 
una ventaja competitiva". Promoviendo un valor agregado más al talento humano denominándolo como activo parte fundamental de una empresa

Según Hernández y Mendieta (2013):

La rotación del personal está influenciada por la desmotivación y la insatisfacción laboral, por lo que se puede afirmar que los motivos que implican la salida de los trabajadores son fundamentalmente laborales y en la mayoría de los casos pueden ser detectados mediante el estudio de la fluctuación potencial, por lo que si se toman las medidas necesarias se puede reducir la rotación real. (p. 838), motivo por el cual deberíamos tomar muy en cuenta esta aseveración en el desarrollo de este artículo llegando a mejorar la productividad de estos actores.

En la medida en que las instituciones sean capaces de incorporar planes de carrera que establezcan procesos de capacitación, opciones de desarrollo humano integral y la integración de equipos de trabajo de alto rendimiento sujetos a procesos de reconocimiento de los logros que sean transparentes y justos, en esa misma medida aumentará la percepción de bienestar de los trabajadores, su motivación y así garantizar una mayor estabilidad de sus colaboradores para disminuir el impacto de la carga laboral, rotación de personal en la productividad. (Hernández y Mendieta, 2013, p.862), el autor hace referencia al trato justo lo que llevara como resultado a la satisfacción plena de los clientes tratados en forma justa y transparente, que van a dar el resultado de mejorar la productividad.

Es muy importante en el desarrollo del presente artículo partir de las conceptualizaciones para lo cual se define a continuación las variables de estudio de la investigación:

\section{Carga Laboral:}

Sanders y McCormick (citado por Patlan, 2013) menciona. "La carga de trabajo es la cantidad de trabajo físico y mental que realiza una persona" (p. 447). La sobrecarga de trabajo ocurre cuando el trabajador percibe que las demandas de trabajo exceden sus habilidades y recursos para cumplir con sus obligaciones laborales de manera exitosa y en un período de tiempo establecido (Veloutsou y Panigyrakis, 2004). Hacer énfasis a este autor toma referencia lo que está sucediendo en la actualidad en las diferentes entidades del sector público.

\section{Rotación de personal:}

"El término de rotación de personal se utiliza regularmente para definir la fluctuación de personal entre una organización y su ambiente" (., Hernández, G y Mendieta, A, 2013, p. 838). Motivo que está sucediendo en la actualidad en la función judicial y que está afectando directamente a la labor diaria y productividad.

\section{Productividad:}

Ruiz (citado por Guerrero y Puerto, 2007) afirma: "Relación existente entre el volumen físico de la producción y los recursos utilizados para obtenerlo" (p. 204). Por otro lado, Tolentino (citado de Marvel, Rodríguez y Núñez, 2014) sugiere:

La productividad es el resultado de la armonía y articulación entre la tecnología, los recursos humanos, la organización y los sistemas, gerenciados o administrados por las personas o eficacia, siempre que se consiga la combinación óptima o equilibrada de los recursos o eficiencia. (p. 555), si hacemos referencia a los autores podemos indicar la relación del estudio en cuanto al desarrollo de las actividades laborales conjunto con su carga horaria y la rotación del personal que estas en muchas ocasiones realizan y que afectan directamente la imagen institucional y productividad en la actualidad. 
ISSN: 2602-8085

Tabla 3. Análisis de productividad.

\begin{tabular}{|c|c|c|c|}
\hline Variable & Dimensiones & Indicador & Subindicador \\
\hline \multirow{7}{*}{$\begin{array}{c}\text { CARGA } \\
\text { LABORAL }\end{array}$} & \multirow{2}{*}{$\begin{array}{l}\text { Carga Física y el } \\
\text { esfuerzo muscular }\end{array}$} & Carga Física Estática & $\begin{array}{l}* \text { Posturas durante el trabajo } \\
* \text { Esfuerzo físico continuo y mantenido durante } \\
\text { cierto tiempo }\end{array}$ \\
\hline & & Carga Física Dinámica & $\begin{array}{l}\text { * Sucesión de tensiones y distensiones de los } \\
\text { músculos activos. } \\
\text { * Gestión y esfuerzos }\end{array}$ \\
\hline & \multirow{3}{*}{ Carga Mental } & Fisiológico & $\begin{array}{l}\text { * Presión sanguínea } \\
* \text { Electroencefalograma } \\
\text { *Frecuencia cardiaca }\end{array}$ \\
\hline & & De Conducta & $\begin{array}{l}* \text { Tiempo de reacción } \\
* \text { Errores } \\
* \text { Olvidos } \\
* \text { Modificaciones de proceso operativo } \\
* \text { Fatiga }\end{array}$ \\
\hline & & Psicológicos & $\begin{array}{l}* \text { Memoria } \\
* \text { Atención } \\
* \text { Coordinación visomotora }\end{array}$ \\
\hline & \multirow{2}{*}{ Carga psíquica } & $\begin{array}{l}\text { Aspectos efectivos referidos } \\
\text { al contenido del trabajo }\end{array}$ & $\begin{array}{l}* \text { Grado de iniciativa } \\
* \text { Grado de ambigüedad } \\
* \text { Grado de cooperación } \\
* \text { Relación con los usuarios, clientes y público. }\end{array}$ \\
\hline & & Carga emocional y afectiva & $\begin{array}{l}* \text { Status social } \\
* \text { Tipo de comunicación } \\
\text { *Niveles de estrés } \\
* \text { Adaptación } \\
\end{array}$ \\
\hline $\begin{array}{l}\text { ROTACION } \\
\text { DE } \\
\text { PERSONAL }\end{array}$ & $\begin{array}{l}\text { *Rotación Voluntaria } \\
\text { *Rotación Involuntaria } \\
\text { *Rotación Positiva } \\
\text { *Rotación Negativa }\end{array}$ & $\begin{array}{l}\text { *Por propia voluntad } \\
\text { *Despido } \\
\text { *Renuncia } \\
\text { *Experiencias del trabajo } \\
\text { *Indecible }\end{array}$ & $\begin{array}{l}\text { *Cuestionarios de Motivación Laboral SL20/23 } \\
\text { *Entrevista de motivación laboral. }\end{array}$ \\
\hline \multirow{6}{*}{ PRODUCTIVIDAD } & Dimensión psicológica & Factores Individuales & $\begin{array}{l}\text { *Motivación } \\
\text { *Competencias } \\
\text { *Satisfacción } \\
\text { *Identificación, compromiso e implicación }\end{array}$ \\
\hline & \multirow{2}{*}{ Dimensión psicosocial } & Factores Grupales & $\begin{array}{l}\text { *Cohesión } \\
* \text { Conflicto } \\
\text { *Participación }\end{array}$ \\
\hline & & Factores Organizacionales & $\begin{array}{l}* \text { Cultura Organizacional } \\
* \text { Liderazgo } \\
* \text { Clima Organizacional }\end{array}$ \\
\hline & \multirow{3}{*}{ Rendimiento Laboral } & Motivación Laboral & $\begin{array}{l}* \text { Satisfacción } \\
\text { *Incentivos }\end{array}$ \\
\hline & & Desempeño Laboral & $\begin{array}{l}\text { *Eficacia } \\
* \text { Eficiencia } \\
\text { *Tiempo }\end{array}$ \\
\hline & & Competencia Laboral & $\begin{array}{l}\text { *Habilidades operativas } \\
\text { *Orientación al logro }\end{array}$ \\
\hline
\end{tabular}


ISSN: 2602-8085

Vol. 3, N³.3, p. 30-43, septiembre, 2019

\section{Metodología}

El tipo de investigación a realizar fue de tipo documental por lo que se revisó la literatura de algunos autores relacionando en una forma las variables de estudio y que fueron abordados por los mismos.

Según Baena (1985) "La investigación documental es una técnica que consiste en la selección y recopilación de información por medio de la lectura crítica de documentos y materiales bibliográficos, de bibliotecas, hemeroteca, centros de documentación e información.", motivo que sustenta mi investigación.

Al respecto Franklin (1997), define a la investigación documental aplicada a la organización de empresas como una técnica de investigación en la que "se deben seleccionar y analizar aquellos escritos que contienen datos de interés relacionados con el estado...", motivo por el cual se realizó esta investigación que tiene como fin la publicación del presente artículo.

\section{Discusión y conclusión.}

Entender la relación existente entre la carga laboral, rotación de personal y como incide en la productividad y satisfacción laboral de los servidores públicos como un determinante de la calidad de vida del individuo es un tema de investigación de interés para el manejo de políticas laborales de un país. Comprender qué aspectos laborales y personales se encuentran asociados a estos factores permite determinar en qué lineamientos de política laboral se debe mejorar.

El trabajo es un derecho de los ciudadanos ecuatorianos y se encuentra garantizado en la Constitución de la República del Ecuador; siendo uno de los deberes más alto del estado, el garantizar todos los derechos humanos y en especial el derecho al trabajo; creando condiciones favorables para que las personas puedan ocuparse dignamente, permitiéndose generar ingresos para el sustento de sus hogares y el mejoramiento de sus condiciones de vida en la sociedad.

Cualquier trabajo conlleva un desgaste, este esfuerzo se denomina carga de trabajo. A diario nuestra mente debe responder a diferentes estímulos o situaciones; recogemos información a través de los sentidos, la procesamos en el cerebro y respondemos con una acción o decisión; a esto lo llamamos carga mental. Todo trabajo mental conlleva un proceso; en primer lugar, la obtención de la información; en segundo lugar, procesamiento la información y por último la toma de decisiones.

Es posible evitar los sobreesfuerzos y sus consecuencias negativas tanto mentales, físicas y afectación sobre la producción laboral. Para ello hay que tomar medidas preventivas en el centro del trabajo, teniendo en cuenta las características de la persona, el diseño del puesto y posibles mejoras, ya sea en el puesto de trabajo y su organización. En el puesto que exige un trabajo estático se debe adoptar medidas que permitan cambiar de postura para evitar el cansancio o realizar varios descansos de corta duración a lo largo de la jornada. También es importante que el puesto de trabajo disponga del espacio suficiente para trabajar con facilidad. Se debe tener en cuenta las condiciones organizativas del trabajo, el tiempo de trabajo, las pausas y descanso, el ritmo, la supervisión del trabajo realizado, el margen de error, etc.

La experiencia y formación son factores claves para prevenir sobreesfuerzos especialmente en casos de carga mental. Así cuando se prevean cambios en los procesos productivos o los equipos de trabajo es muy importante planificar con antelación planes de formación. Se conoce que cualquier trabajo exige en mayor o menor medida un esfuerzo físico o metal. El plan de prevención de la empresa no debe obviar esta realidad y tanto en la evaluación de riesgos como en la planificación de las actividades preventivas, debe reflejarse los riesgos para la salud relacionada 
con la carga física y mental del trabajo. El trabajo seguro y saludable es un derecho, participar en la búsqueda de mejores condiciones de trabajo, es un deber del estado y de los principales directivos de las instituciones públicas, pues de eso mucho depende disminuir la rotación de personal e incrementar la productividad laboral dentro del sector público.

Concluir que el estudio y análisis en la toma de decisiones tanto en la carga horaria asignada, rotación del personal son factores que van afectar directamente a la productividad de los trabajadores de este sector productivo del estado.

\section{Bibliografía}

Andrade, J., Sanabria, Y., Morales, M., Rodríguez, J y Oyuela, J. (2013). Prevalencia del síndrome de Burnout en personal de enfermería de una clínica de la ciudad de Ibagué. Psicogente, 16(19), 170-183.

Aranda, C., Pando, M y Salazar. (2016) Confiabilidad y validación de la escala Maslach Burnout Inventory (Hss) en trabajadores del occidente de México. Salud Uninorte, Vol.32 (2), 218-227.

Beek, I., Hu, Q y Wilmar, B. (2012). For Fun, Love, or Money: ¿What Drives Workaholic, Engaged, and Burned-Out Employees at Work? Applied Psychology, 61(1), 30-55.

Ceballos, P., Rolo, G., Hernández, E., Díaz, D., Paravic, T y Burgos, M. (2015) Factores psicosociales y Carga Mental de Trabajo: Una realidad percibida por enfermeras/os en Unidades Críticas. Rev. Latino-Am. Enfermagem, 23(2), 315-322.

Ceballos, P., Rolo, G., Hernández, E., Díaz, D., Paravic, T y Burgos, M. (2016) Validación de la Escala Subjetiva de Carga mental de Trabajo (ESCAM) en profesionales de la salud de Chile: Universitas Psychologica, 15(1), 261-270.

Ceballos, P., Rolo, G., Hernández, E., Díaz, D., Paravic, T y Burgos, M. (2016). Factores psicosociales y carga mental de trabajo: una realidad percibida por enfermeras/os en Unidades Críticas Latino Americana de Enfermagen, 23 (2), 315-322.

Chaparro, D., Guzmán, A., Naizaque, L., Ortiz, S., Jiménez y Giovanni, W. (2015). Factores que originan la rotación del personal auxiliar de odontología. Universitas Odontológica, 34(72), 75-82.

Cruz, M., Chávez, M., Barcellos, R., Da Silva, L., Oliveira, L y Pedrao, L. (2010). Exceso de trabajo y agravios mentales a los trabajadores de la salud. Revista Cubana de Enfermería, 26(1), 52-64.

González Muñoz, E L; Gutiérrez Martínez, R E; (2006). La carga de trabajo mental como factor de riesgo de estrés en trabajadores de la industria electrónica. Revista Latinoamericana de Psicología, 38() 259-270.

Guerrero, J y Puerto, Y. (2007). Productividad, trabajo y salud: la perspectiva psicosocial. Revista Colombiana de Psicología, Núm. 16, 203-234.

Hakanen, J y Schaufeli, W. (2012). ¿Do burnout and work engagement predict depressive symptoms and rife satisfacción? A three-wave seven-year prospective study. Journal of Affective Disorders, 141(1), 415-424.

Heinemann, L y Heinemann, T. (2017). Burnout Research: Emergence and Scientific Investigation of a Contested Diagnosis. Sage Open, 7(1), 1-12

Hernández Chávez, Y.; Hernández Chávez, G. y Mendieta Ramírez, A. (2013) Modelo de rotación de personal y prácticas organizacionales. Historia y Comunicación Social. Vol. 18. N. ${ }^{\circ}$ Especial Diciembre. Págs. 837-863. 
Hernández, F y Pena, I. (2008). Efectividad de la estrategia de recursos humanos: Modelo integrador de la teoría de recursos y capacidades y la teoría del comportamiento en las entidades financieras de la economía social. REVESCO: Revista de Estudios Cooperativos, (94), 27 -58.

Ibáñez, J., López, J., Márquez, A., Sánchez, N., Flores, L y Vera, A. (2012). Variables sociodemográficas relacionadas al Síndrome de Burnout en docentes de colegios distritales. Psicología: Avances de la Disciplina, 6(2), 103-116.

Marenco, A y Ávila, J. (2016). Burnout y problemas de salud mental en docentes: diferencias según características demográficas y sociolaborales. Psychologia: Avances de la Disciplina, 10(1), 91-100.

Marvel, M., Rodríguez, C y Núñez, M. (2011). La productividad desde una perspectiva humana: Dimensiones y factores. Intangible Capital, $7(2), \quad 549-584 . \quad$ DOI: http://hdl.handle.net/2099/11316

Patlán, J. (2013). Efecto del burnout y la sobrecarga en la calidad de vida en el trabajo. Estudios Gerenciales, 29 (129) 445-455.

Polo, J. D., Santiago, V., Navarro, M. C. \& Alí, A. (2016). Creencias irracionales, síndrome de Burnout y adicción al trabajo en las organizaciones. Psicogente, 19(35), 148-160. DOI: http://doi.org/10.17081/psico.19.35.1215

Rodríguez, M. y Dabos, G. (2017). Gestión individual del equilibrio entre el trabajo y la vida personal. Revista de la Facultad de Ciencias Económica, XXV (1), 219-242. DOI: http://dx.doi. org/10.18359/rfce.1055

Van, C., Peters, M.., Schaufeli, W. (2014). Enough is enough: Cognitive antecedents of Workaholism and its aftermath. Human Resource Managemen, 53(157-177)

Vargas, L., Niño, C y Acosta, Y. (2017) Estrategias que modulan el síndrome de Burnout en enfermeros (as). Revista Ciencia y Cuidado, 14(1), 111-131.

Vargas, Z. (2009). La investigación aplicada: Una forma de conocer las realidades con evidencias científica. Revista educación, 33(1), 155 - 165.

Zenteno-Hidalgo, A. C., \& Durán Silva, C. A. (2016). Factores y prácticas de alto desempeño que influyen en el clima laboral: análisis de un caso. Innovar, 26(59), 119-136. DOI: http://dx.doi.org/10.15446/innovar.v26n59.54367

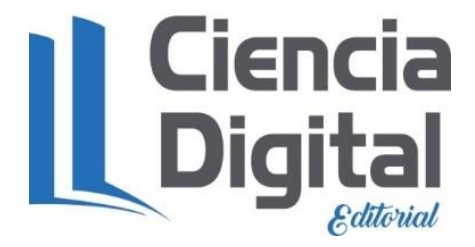




\section{PARA CITAR EL ARTÍCULO INDEXADO.}

Maliza Cerezo, G., \& Bastidas Vaca, C. (2019). Efecto de la carga laboral y la rotación del personal en la productividad de los servidores judiciales en el Consejo de la Judicatura de Los Ríos, Ecuador en el año 2018. Ciencia Digital, 3(3.3), 30-43. https://doi.org/10.33262/cienciadigital.v3i3.3.768

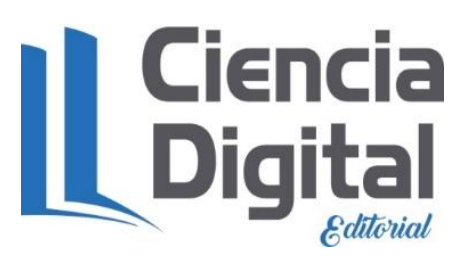

El artículo que se publica es de exclusiva responsabilidad de los autores y no necesariamente reflejan el pensamiento de la Revista Ciencia Digital.

El artículo queda en propiedad de la revista y, por tanto, su publicación parcial y/o total en otro medio tiene que ser autorizado por el director de la Revista Ciencia Digital.
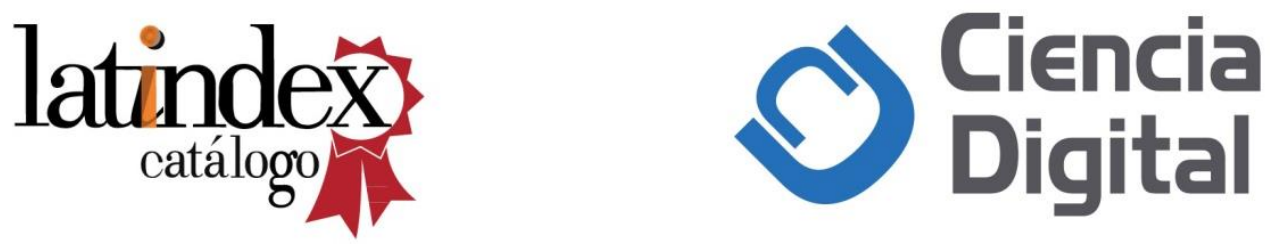